\title{
Causality between Research Output and Economic Growth in BRICS
}

\author{
Roula Inglesi-Lotz ${ }^{1}$ - Tsangyao Chang ${ }^{2} \cdot$ Rangan Gupta $^{1}$ \\ ${ }^{1}$ Department of Economics, University of Pretoria, Pretoria, South Africa \\ ${ }^{2}$ Department of Finance, Feng Chia University, Taichung, Taiwan \\ Roula Inglesi-Lotz (Corresponding author) \\ Email: roula.inglesi-lotz@up.ac.za \\ Tsangyao Chang \\ Email: tychang@fcu.edu.tw \\ Rangan Gupta \\ Email: rangan.gupta@up.ac.za
}

\begin{abstract}
This paper examines the causal relationship between the economic growth and research output of the BRICS countries (i.e., Brazil, Russia, India, China, and South Africa) for the period 1981-2011. Essentially this study looks at the human quality demonstrated by the production of knowledge (published papers) and how it gets affected and influences the economic growth of these countries. BRICS are among the fastest growing emerging economies that are grouped together in this study. Using panel causality analysis techniques, we account for cross-section dependency and heterogeneity among them. Our empirical results support no causality in any direction between research papers as a percentage share to the world and economic growth for all the BRICS, with the exception of India, for which the feedback hypothesis is confirmed. Our findings provide important policy implications for research policies and strategies for BRICS countries.
\end{abstract}

Keywords: Research Papers Published; Economic Growth; BRICS; Dependency and Heterogeneity; Panel Causality Test

\section{Introduction}

The improvement of human capital demonstrated through the accumulation of 
knowledge and its impact to the economic growth of the countries is not a recent topic of discussion in the literature (Romer 1986; Lucas 1988; Tamura 1991; Schumpeter 2000). It is widely acknowledged that knowledge externalities are proven positive from a microeconomic point of view (i.e. a given firm is more productive the higher the average knowledge stock in the sector is) but also looking at the economy in its entirety. Knowledge, as Schumpeter (2000) can be incorporated in the productive activities of a country through two main concepts: invention (the discovery of new knowledge) and innovation (the introduction of new methods, products and approaches to the industry).

A number of applied studies (Price 1978; Kealey 1996; De Moya-Anego and Herrero-Solana 1999; King 2004; Fedderke and Schirmer 2006; Lee et al. 2011; Shelton and Leydersdorff 2011; Inglesi-Lotz and Pouris 2013) have investigated the relationship between economic growth and accumulated knowledge. They have identified that the relationship can imply causality running from any of the two indicators (growth and accumulated knowledge) to the other: for example, higher levels of economic growth in a country can stimulate higher knowledge capacities and better quality of human capital while on the other side, as in traditional endogenous economic growth theory, better human capital and higher levels of knowledge accumulation can promote increasing economic growth in countries. Lee et al. (2001) have concluded that the direction and strength of the relationship depends on the developmental stage of the countries: they found lack of relationship between economic growth and accumulated knowledge measured in research performance for the developed economies of their sample while strong relationship for the developing.

In this paper, we group together five of the most important and fastest growing developing countries (i.e., Brazil, Russia, India, China, and South Africa (BRICS)) and examine whether accumulated knowledge and improved human capital has a role to play in their path to higher economic growth or whether their increasing growth and development affects the quality of human capital in their economies. In other words, the particular aim of 
the paper is to re-investigate the causal relationship between research papers published in terms of their world share and economic growth in BRICS countries over the period of 1981-2011. To the best of our knowledge, this paper is the first paper to examine the relationship in the case of BRICS countries. Two studies related to our work are the papers by Lee et al. (2011) and Inglesi-Lotz and Pouris (2013). While Inglesi-Lotz and Pouris (2013) looks at causality between research output and growth for single country, namely South Africa, Lee et al., (2010) looks at 25 countries individually, including Brazil, China and India. Inglesi-Lotz and Pouris (2013) concluded that causality runs from research output to growth in South Africa, and Lee et al. (2011) suggested that there is bi-directional causality in Brazil, unidirectional causality from GDP to research in China, and unidirectional causality from research to GDP in India.

We however believe that our approach is methodologically superior than single country analyses, especially when considering similar, in our case emerging economies, as we allow not only for heterogeneity but also cross-sectional dependence. The importance of the latter is paramount, simply because of the inter-relatedness of these countries, not only in terms of their policies, but also co-movement of the key variables. Specifically, we examine the existence of cross-sectional dependency and heterogeneity across these countries. Ignoring cross-section dependency leads to substantial bias and size distortions (Pesaran, 2006), implying that testing for the cross-section dependence is a crucial step in a panel data analysis. Also, even though we use a panel-setting and hence acquire efficiency in terms of more available data, our approach allows for a country-specific analysis, controlling for cross-sectional interrelations and country-specific heterogeneity. Note that, since these single country analyses use around 30 or less observations in a vector autoregressive framework, the available degrees of freedom are quite small, and hence, the results are likely to suffer from small-sample bias, unless some bootstrapping procedures are used to obtain critical values for the tests. Further, our approach, does not require us to difference variables of interest to 
ensure stationarity, and hence, does not suffer from possible biasedness in results that are likely due to such transformations.

Measuring economic growth is standard in the literature by using indicators such as Gross Domestic Product (GDP) or GDP per capita. On the contrary, finding the appropriate indicator to represent accumulated knowledge is a complex and difficult task. Traditional methods use the total secondary school enrolments in a country as an indication of human capital. Studies such as the one by Fedderke and Schirmer (2006) have used input indicators for the measurement of the stock of knowledge, for example the Research and Development (R\&D) expenditures of the country. To measure a country's research performance, other studies (De Moya-Anego and Herrero-Solana 1999; King 2004; Inglesi-Lotz and Pouris 2013) have preferred output indicators that are primarily been borrowed by the field of scientometrics. Inglesi-Lotz and Pouris (2013) discuss that output indicators are more robust than input and convey more information: for example R\&D allocated funds might be spent without any significant contribution to the R\&D of the country.

In this paper, we choose to employ scientometric indicators, such as the number of academic papers published in a country, following the conclusions of Pouris and Pouris (2009) that consider scientometric analysis as one of the most objective and straightforward ways of measuring innovation and research performance of a country, a region or an institution. On top of that, Price (1978) states that "for those who are working at the research front, publication is not just an indicator but in, a very strong sense, the end product of their creative effort". For comparison purposes, the research publications of a country or an institution are considered good proxies of scientific manpower and knowledge accumulation (Schubert and Telcs 1986). This is especially helpful in an analysis of developing countries, such as the BRICS, that lack sophisticated monitoring mechanisms of science, technology and innovation. In addition, research is thus measured by the same standard, i.e., the research output in ISI accredited journals; a measure easily comparable. More specifically, as in 
Inglesi-Lotz and Pouris (2013) research output is measured by the comparative research performance of the country in relation to the rest of the world in terms of number of publications.

The rest of the paper is organized as follows. Section 2 discusses in detail the methodology of the bootstrap panel Granger causality test proposed by Kónya (2006). Section 3 presents the empirical results and finally in section 4 we discuss some the policy implications of our empirical findings.

\section{Methodology and data}

\subsection{Preliminary Analysis}

One important issue in a panel causality analysis is to take into account possible cross-section dependence across countries. This is because high degree of globalization, international trade and financial integration make a country to be sensitive to the economics shocks in other countries. Cross-sectional dependency may play important role in detecting causal linkages for BRICS countries since these countries are highly integrated.

The second issue to decide before carrying out causality tests is to find out whether the slope coefficients are treated as homogenous and heterogeneous to impose causality restrictions on the estimated parameters. As pointed out by Granger (2003), the causality from one variable to another variable by imposing the joint restriction for the panel is the strong null hypothesis Furthermore, as Breitung (2005) contends the homogeneity assumption for the parameters is not able to capture heterogeneity due to country specific characteristics. In the research papers published and economic growth nexus - as in many economic relationships - while there may be a significant relationship in some countries, vice versa may also be true in some others.

Given the above consideration before we conduct tests for causality, we start with testing for cross-sectional dependency, followed by slope homogeneity across countries. Then, we 
decide to which panel causality method should be employed to appropriately determine the direction of causality between research papers published and economic growth in BRICS countries. In what follows, we outline the essentials of econometric methods used in this study.

\subsubsection{Testing cross-section dependence}

To test for cross-sectional dependency, the Lagrange multiplier (LM hereafter) test of Breusch and Pagan (1980) has been extensively used in empirical studies. The procedure to compute the LM test requires the estimation of the following panel data model:

$$
y_{i t}=\alpha_{i}+\beta_{i}^{\prime} x_{i t}+u_{i t} \text { for } i=1,2, \ldots, N ; t=1,2, \ldots, T
$$

where $i$ is the cross section dimension, $t$ is the time dimension, $x_{i t}$ is $k \times 1$ vector of explanatory variables, $\alpha_{i}$ and $\beta_{i}$ are respectively the individual intercepts and slope coefficients that are allowed to vary across states.

In the LM test, the null hypothesis of no-cross section dependence- $H_{0}: \operatorname{Cov}\left(u_{i t}, u_{j t}\right)=0$ for all $t$ and $i \neq j$ - is tested against the alternative hypothesis of cross-section dependence $H_{1}: \operatorname{Cov}\left(u_{i t}, u_{j t}\right) \neq 0$, for at least one pair of $i \neq j$. In order to test the null hypothesis, Breusch and Pagan (1980) developed the LM test as:

$$
L M=T \sum_{i=1}^{N-1} \sum_{j=i+1}^{N} \hat{\rho}_{i j}^{2}
$$

where $\hat{\rho}_{i j}$ is the sample estimate of the pair-wise correlation of the residuals from Ordinary Least Squares (OLS) estimation of equation (1) for each $i$.

Under the null hypothesis, the $L M$ statistic has asymptotic chi-square with 
$N(N-1) / 2$ degrees of freedom. It is important to note that the $\mathrm{LM}$ test is valid for $\mathrm{N}$ relatively small and $\mathrm{T}$ sufficiently large. However, the $C D$ test is subject to decreasing power in certain situations that the population average pair-wise correlations are zero, although the underlying individual population pair-wise correlations are non-zero (Pesaran et al., 2008, p.106). Furthermore, in stationary dynamic panel data models the CD test fails to reject the null hypothesis when the factor loadings have zero mean in the cross-sectional dimension. In order to deal with these problems, Pesaran et al. (2008) proposes a bias-adjusted test which is a modified version of the LM test by using the exact mean and variance of the LM statistic. The bias-adjusted LM test is:

$$
L M_{a d j}=\sqrt{\left(\frac{2 T}{N(N-1)}\right)} \sum_{i=1}^{N-1} \sum_{j=i+1}^{N} \hat{\rho}_{i j} \frac{(T-k) \hat{\rho}_{i j}^{2}-\mu_{T i j}}{\sqrt{v_{T i j}^{2}}}
$$

where $\mu_{T i j}$ and $v_{T i j}^{2}$ are respectively the exact mean and variance of $(T-k) \hat{\rho}_{i j}^{2}$, that are provided in Pesaran et al. (2008, p.108). Under the null hypothesis with first $\mathrm{T} \rightarrow \infty$ and then $\mathrm{N} \rightarrow \infty, L M_{a d j}$ test is asymptotically distributed as standard normal.

\subsubsection{Testing slope homogeneity}

Second issue in a panel data analysis is to decide whether or not the slope coefficients are homogenous. The causality from one variable to another variable by imposing the joint restriction for whole panel is the strong null hypothesis (Granger, 2003). Moreover, the homogeneity assumption for the parameters is not able to capture heterogeneity due to country specific characteristics (Breitung, 2005).

The most familiar way to test the null hypothesis of slope homogeneity- $H_{0}: \beta_{i}=\beta$ for all $i$ - against the hypothesis of heterogeneity- $H_{1}: \beta_{i} \neq \beta_{j}$ for a non-zero fraction of 
pair-wise slopes for $i \neq j$ - is to apply the standard $F$ test. The $F$ test is valid for cases where the cross section dimension $(\mathrm{N})$ is relatively small and the time dimension $(\mathrm{T})$ of panel is large; the explanatory variables are strictly exogenous; and the error variances are homoscedastic. By relaxing homoscedasticity assumption in the $F$ test, Swamy (1970) developed the slope homogeneity test on the dispersion of individual slope estimates from a suitable pooled estimator. However, both the $F$ and Swamy's test require panel data models where $N$ is small relative to $T$ [24]. Pesaran and Yamagata (2008) proposed a standardized version of Swamy's test (the so-called $\tilde{\Delta}$ test) for testing slope homogeneity in large panels. The $\tilde{\Delta}$ test is valid as $(N, T) \rightarrow \infty$ without any restrictions on the relative expansion rates of $\mathrm{N}$ and $\mathrm{T}$ when the error terms are normally distributed. In the $\tilde{\Delta}$ test approach, first step is to compute the following modified version of the Swamy's test:

$$
\tilde{S}=\sum_{i=1}^{N}\left(\widehat{\beta}_{i}-\tilde{\beta}_{W F E}\right)^{\prime} \frac{x_{i}^{\prime} M_{\tau} x_{i}}{\tilde{\sigma}_{i}^{2}}\left(\widehat{\beta}_{i}-\tilde{\beta}_{W F E}\right)
$$

where $\hat{\beta}_{i}$ is the pooled OLS estimator, $\tilde{\beta}_{W F E}$ is the weighted fixed effect pooled estimator, $M_{\tau}$ is an identity matrix, the $\tilde{\sigma}_{i}^{2}$ is the estimator of $\sigma_{i}^{2} \cdot{ }^{1}$ Then the standardized dispersion statistic is developed as:

$$
\tilde{\Delta}=\sqrt{N}\left(\frac{N^{-1} \tilde{S}-k}{\sqrt{2 k}}\right)
$$

Under the null hypothesis with the condition of $(N, T) \rightarrow \infty$ so long as $\sqrt{N} / T \rightarrow \infty$ and the error terms are normally distributed, the $\tilde{\Delta}$ test has asymptotic standard normal distribution.

\footnotetext{
${ }^{1}$ In order to save space, we refer to Pesaran and Yamagata (2008) for the details of estimators and for Swamy's test.
} 
The small sample properties of $\tilde{\Delta}$ test can be improved under the normally distributed errors by using the following bias adjusted version:

$$
\tilde{\Delta}_{a d j}=\sqrt{N}\left(\frac{N^{-1} \tilde{S}-E\left(\tilde{z}_{i t}\right)}{\sqrt{\operatorname{var}\left(\tilde{z}_{i t}\right)}}\right)
$$

where the mean $E\left(\tilde{z}_{i t}\right)=k$ and the variance $\operatorname{var}\left(\tilde{z}_{i t}\right)=2 k(T-k-1) / T+1$.

\subsection{Panel Causality Test}

Once the existence of cross-section dependency and heterogeneity across BRICS countries is ascertained, we apply a panel causality method that should account for these dynamics. The bootstrap panel causality approach proposed by Kónya (2006) is able to account for both cross-section dependence and country-specific heterogeneity. This approach is based on Seemingly Unrelated Regression (SUR) estimation of the set of equations and the Wald tests with individual specific country bootstrap critical values. Since country-specific bootstrap critical values are used, the variables in the system do not need to be stationary, implying that the variables are used in level form irrespectively of their unit root and cointegration properties. Thereby, the bootstrap panel causality approach does not require any pre-testing for panel unit root and cointegration analyses. Besides, by imposing country specific restrictions, we can also identify which and how many states exist in the Granger causal relationship.

The system to be estimated in the bootstrap panel causality approach can be written as: 


$$
\begin{aligned}
& y_{1, t}=\alpha_{1,1}+\sum_{i=1}^{l y_{1}} \beta_{1,1, i} y_{1, t-i}+\sum_{i=1}^{l x_{1}} \delta_{1,1, i} x_{1, t-i}+\varepsilon_{1,1, t} \\
& y_{2, t}=\alpha_{1,2}+\sum_{i=1}^{l y_{1}} \beta_{1,2, i} y_{2, t-i}+\sum_{i=1}^{l x_{1}} \delta_{1,2, i} x_{2, t-i}+\varepsilon_{1,2, t} \\
& \vdots \\
& y_{N, t}=\alpha_{1, N}+\sum_{i=1}^{l y_{1}} \beta_{1, N, i} y_{N, t-i}+\sum_{i=1}^{l x_{1}} \delta_{1, N, i} x_{1, N, t-i}+\varepsilon_{1, N, t}
\end{aligned}
$$

and

$$
\begin{aligned}
& x_{1, t}=\alpha_{2,1}+\sum_{i=1}^{l y_{2}} \beta_{2,1, i} y_{1, t-i}+\sum_{i=1}^{l x_{2}} \delta_{2,1, i} x_{1, t-i}+\varepsilon_{2,1, t} \\
& x_{2, t}=\alpha_{2,2}+\sum_{i=1}^{l y_{2}} \beta_{2,2, i} y_{2, t-i}+\sum_{i=1}^{l x_{2}} \delta_{2,2, i} x_{2, t-i}+\varepsilon_{2,2, t} \\
& \vdots \\
& x_{N, t}=\alpha_{2, N}+\sum_{i=1}^{l y_{2}} \beta_{2, N, i} y_{N, t-i}+\sum_{i=1}^{l x_{2}} \delta_{2, N, i} x_{N, t-i}+\varepsilon_{2, N, t}
\end{aligned}
$$

Where $y$ denotes real income, $x$ refers to research papers published (in terms of total number of papers published or percentage world share), $l$ is the lag length.

Since each equation in this system has different predetermined variables while the error terms might be contemporaneously correlated (i.e. cross-sectional dependency), these sets of equations are the SUR system.

In the bootstrap panel causality approach, there are alternative causal linkages for each country in the system that (i) there is one-way Granger causality from $x$ to $y$ if not all $\delta_{1, \mathrm{i}}$ are zero, but all $\beta_{2, \mathrm{i}}$ are zero, (ii) there is one-way Granger causality running from $y$ to $x$ if all $\delta_{1, \mathrm{i}}$ are zero, but not all $\beta_{2, \mathrm{i}}$ are zero, (iii) there is two-way Granger causality between $x$ and $y$ if neither $\delta_{1, i}$ nor $\beta_{2, \mathrm{i}}$ are zero, and finally (iv) there is no Granger causality in any direction between $x$ and $y$ if all $\delta_{1, \mathrm{i}}$ and $\beta_{2, \mathrm{i}}$ are zero.

\subsection{Data}

The annual data used in this study cover the period from 1981 to 2011 for the BRICS 
countries. Economic growth is proxied by total real GDP (RGDP) measured in constant 2005 U.S. dollars and comes from the World Development Indicators (WDI 2011). Research papers published (in terms of percentage share to the world) are derived from the Institute of Scientific Information (ISI) Thomson Reuters family of databases. In the National Science Indicators database, the following research outputs are counted: articles, notes, reviews and proceeding papers; however letters, editorials, corrections and mere abstracts are not taken into consideration (Inglesi-Lotz and Pouris, 2011). Although other databases were also used in the literature, the ISI database is considered the best available option due to its multidisciplinary and interdisciplinary character. It is important to note here that the database covers the most significant journals in the world in all fields (approximately 6, 000 peer-reviewed journals) to ensure a certain level of quality in research outputs.

Table 1. Summary statistics of GDP

\begin{tabular}{lrrrrrrl}
\hline country & Mean & Max. & Min. & Std. Dev. & Skew. & Kurt. & J.-B. \\
\hline Brazil & 709139 & 107305 & 459357 & 174249 & 0.524 & 2.319 & 2.016 \\
Russia & 725759 & 941711 & 482625 & 144167 & -0.253 & 1.779 & 2.255 \\
India & 571650 & 1354422 & 212280 & 326049 & 0.920 & 2.795 & 4.431 \\
China & 1396872 & 4222640 & 228164 & 1154025 & 1.028 & 2.956 & $5.471^{*}$ \\
South Africa & 198547 & 307075 & 148188 & 49809 & 0.867 & 2.394 & 4.361 \\
\hline
\end{tabular}

Note: The sample period is from 1981 to 2011. * denotes that the series confirms the null hypothesis of "No normality" at the $10 \%$ level of significance. Std.Dev=standard deviation; Skew= skewness; Kurt=kurtosis, J.-B= Jarque-Bera test.

Table 2. Summary statistics of \% world share of papers published

\begin{tabular}{lccccccc}
\hline & Mean & Max. & Min. & Std. Dev. & Skew. & Kurt. & J.-B. \\
\hline Brazil & 1.22 & 2.75 & 0.44 & 0.77 & 0.78 & 2.35 & 3.73 \\
Russia & 3.91 & 5.53 & 2.26 & 1.07 & -0.09 & 1.72 & 2.16 \\
India & 2.65 & 3.67 & 2.09 & 0.42 & 0.96 & 3.03 & $4.76^{*}$ \\
China & 3.59 & 11.87 & 0.30 & 3.45 & 1.09 & 2.87 & $6.23^{* *}$ \\
South Africa & 0.53 & 0.67 & 0.47 & 0.06 & 0.81 & 2.53 & 3.71 \\
\hline
\end{tabular}

Note: See Notes to Table 1. *[**] denotes that the series confirms the null hypothesis of "No normality" at the $10 \%$ [5\%] level of significance. 
Based on the data (see Table 1 and 2), China and South Africa have the highest and lowest of mean real GDP of US\$1,396,872 and US\$198,547 million of dollars, respectively, Russia and South Africa have the highest and lowest percentage world share of papers published of 3.91 and 0.53, respectively, and China and South Africa have the highest and lowest total mean number of papers published of 33,997.4 and 4037.1, respectively. Most of the data series are approximately normal, with the exception of real GDP for China and \% world share of papers published for India and China.

\section{Empirical findings}

Before we test for causality we first test for both cross-sectional dependency and country-specific heterogeneity as we believe that BRICS countries are highly integrated in their economic relations. To investigate the existence of cross-section dependence we carried out four different tests $\left(L M, \mathrm{CD}_{\mathrm{lm}}, \mathrm{CD}, \mathrm{LM}_{\mathrm{adj}}\right)$. Secondly, as indicated by Kónya (2006), the selection of optimal lag structure is of importance because the causality test results may depend critically on the lag structure. In determining lag structure we follow Kónya's approach that maximal lags are allowed to differ across variables, but to be same across equations. We estimate the system for each possible pair of $l y_{1}, l x_{1}, l y_{2}$ and $l x_{2}$ respectively by assuming from 1 to 4 lags and then choose the combinations which minimize the Schwarz Bayesian Criterion.

Tests for cross-sectional dependency and heterogeneity are presented in Table 3. As can be seen, it is clear that the null hypothesis of no cross-sectional dependency and slope heterogeneity across the countries is strongly rejected at the conventional levels of significance (1\% or $10 \%)$. This finding implies that a shock that occurred in one of these BRICS countries seems to be transmitted to other countries. Furthermore, the rejection of slope homogeneity implies that the panel causality analysis by imposing homogeneity 
Table 3. Cross-sectional Dependence and Homogeneous Tests (\% world share)

\begin{tabular}{lc}
\hline Test & statistic \\
\hline LM & $19.52^{* *}$ \\
$C D_{L M}$ & $2.13^{* *}$ \\
$C D$ & $2.96 * *$ \\
$L M_{a d j}$ & $23.119 * * *$ \\
$\tilde{\Delta}$ & $172.49 * * *$ \\
$\tilde{\Delta}$ & \\
\hline
\end{tabular}

Note: $1 . * * *$ and $*$ indicate significance at the 0.01 and 0.1 levels, respectively.

restriction on the variable of interest results in misleading inferences. In this respect, the panel causality analysis based on estimating a panel vector autoregression and/or panel vector error correction model by means of generalized method of moments and of pooled ordinary least square estimator is not appropriate approach in detecting causal linkages between research papers published (percentage share to the world) and economic growth in BRICS countries.

Table 4: Research papers (\% world share) does not Granger cause GDP

\begin{tabular}{lllcc}
\hline & & \multicolumn{3}{c}{ Bootstrap Critical Value } \\
\hline & & $10 \%$ & $5 \%$ & $1 \%$ \\
\hline Brazil & 0.410 & 4.692 & 6.838 & 12.839 \\
Russia & 2.675 & 5.255 & 7.643 & 15.735 \\
India & $\mathbf{4 . 2 5 7 *}$ & 3.935 & 5.756 & 10.479 \\
China & 1.723 & 4.818 & 6.796 & 12.590 \\
South Africa & 0.107 & 4.080 & 5.879 & 10.197 \\
\hline
\end{tabular}

Note: ** indicates significance at the 0.05 level.

2. Bootstrap critical values are obtained from 10,000 replications. 
Table 5: GDP does not Granger cause Research papers (\% world share)

\begin{tabular}{lcccc}
\hline & & \multicolumn{3}{c}{ Bootstrap Critical Value } \\
\cline { 3 - 5 } & & $10 \%$ & $5 \%$ & $1 \%$ \\
\hline Brazil & 6.116 & 9.863 & 13.103 & 22.004 \\
Russia & 0.439 & 6.032 & 8.839 & 15.818 \\
India & $\mathbf{1 5 . 0 0 8 * *}$ & 10.872 & 14.347 & 23.172 \\
China & 2.749 & 11.654 & 15.198 & 23.913 \\
South Africa & 2.264 & 9.600 & 12.808 & 20.396 \\
\hline
\end{tabular}

Note: $1 . * * *$ and $* *$ indicate significance at the 0.01 and 0.05 respectively.

2. Bootstrap critical values are obtained from 10,000 replications.

The establishment of the existence of cross-sectional dependency and heterogeneity across BRICS countries suggests the suitability of the bootstrap panel causality approach. The results of the bootstrap causality tests are presented in Tables 4 and 5 .

Except for India, we find no causal relationship between the research papers in terms of percentage share to the world and economic growth to any of the other four countries. In India there was a bidirectional causality running between research papers published (in terms of both percentage world share and number of papers published) and economic growth thus supporting the feedback hypothesis where research papers published and GDP serve as complements to each other. The policy implication of our finding is that reduced research papers published may lead to adverse effects on economic growth in India.

\section{Conclusions}

This study applied the bootstrap panel Granger causality approach to test the causal link between accumulated knowledge measured as the research performance of the country an proxied by the research papers of a country as a percentage share to the world and economic growth using data from the BRICS countries (i.e., Brazil, Russia, India, China, and South 
Africa) over the period of 1981-2011. Regarding the research-economic growth nexus, our empirical results support the neutrality hypothesis for Brazil, Russia, China and South Africa and the feedback hypothesis for India.

The confirmation of feedback hypothesis is of utmost importance for the Indian policy makers. The country has been considered a "destination for IT and business process outsourcing" (Wharton, n.d.) and as a main center for pioneering R\&D projects for not only local but also international organisations such as Microsoft and Motorola. The industries that prefer India as the new emerging R\&D destination vary from pharmaceuticals to telecommunications. Our findings propose that even higher investment towards $R \& D$ activities and academic institutions will promote further the positive circle from higher economic growth to even better research performance and improved human capital and vice versa.

On one hand, for South Africa, Inglesi-Lotz and Pouris (2013) found that the percentage share of academic papers to the world causes economic growth in the country for the period 1980 to 2008 . On the other hand, Lee et al. (2011) suggested that there is bi-directional causality in Brazil, unidirectional causality from GDP to research in China, and unidirectional causality from research to GDP in India. Here the results show that there is a no causal relationship between the two for Brazil, China and South Africa, while, bi-directional causality exists for India. The dissimilarity of the results could be attributed to two reasons. In general, causality analyses are known to be methodology-dependent. The methodology used here tends to be more robust than single-country analysis, simply because it allows for cross-sectional dependence, which is of tremendous importance here given the inter-relatedness of the countries in terms of their economic policies and co-movements of key variables; not accounting for which are likely to lead to biased results. Note that this is more so the case, given the two variables under consideration are real GDP and number of papers in a specific country as percentage of total number of papers in the world are surely 
related to each other for the cross-section. The difference in the findings might also be dependent on the time period of the analysis (here, we add the years 2009-2011 for South Africa and 2008-2011 for Brazil, China, India). In these years, the global economic crisis had serious impact on the country's economy, investment choices and R\&D expenditures. Also note that, unlike Lee et al. (2011), we use real GDP instead of nominal GDP, and research papers published as a percentage share to the world instead of total number of papers published by a specific country.

Overall, the main conclusion here is that the research performance does not affect or get affected by economic growth for all the BRICS countries, with the exception of India. Hence, although the BRICS countries present certain similar characteristics and ideas of improving the global economic situation, their policies regarding the investment on R\&D activities should not be homogeneous.

\section{References}

Breitung, J.: A parametic approach to the estimation of cointegration vectors in panel data. Econometric Reviews 24, 151-173 (2005).

Breusch, T. S., and Pagan, A. R. : The Lagrange Multiplier test and its applications to model specification in econometrics. The Review of Economic Studies, 47(1), 239-253 (1980).

De Moya-Anegón, F. and Herrero-Solana, V.: Science in America Latina: A comparison of bibliometric and scientific-technical indicators. Scientometrics 46:299-320(1999).

Fedderke, J.W. and Schirmer, S.; The R\&D performance of the South African manufacturing sector, 1970-1983. Economic Change 39:125-151(2006).

Granger, CWJ. :Some aspects of causal relationships. Journal of Econometrics 112, 69-71 (2003). 
Inglesi-Lotz, R. and Pouris, A.: The influence of scientific research output of academics on economic growth in South Africa: an autoregressive distributed lag (ARDL) application. Scientometrics 95 (1): 129-139 (2013).

Kealey, T.; The Economic Laws of Scientific Research. New York: St. Martin's Press (1996). King, D.A.: The scientific impact of nations. What different countries get for their research spending. Nature 430:311-316 (2004).

Kónya, L.: Exports and growth: granger causality analysis on OECD countries with a panel data approach. Economic Modelling, 23, 978-992 (2006).

Lee, L-C., Lin, P-H., Chuang, Y-W. and Lee, Y-Y.: Research output and economic productivity: a Granger causality test. Scientometrics 89:465-478 (2011).

Lucas, R.E.: On the mechanics of economic development. Journal of Monetary Economics 22:3-42 (1988)

Pesaran, M. H.: Estimation and Inference in Large Heterogeneous Panels with Multifactor Error Structure. Econometrica. 74 (4), 967-1012 (2006).

Pesaran, M. H., Ullah, A., Yamagata, T.: A bias-adjusted LM test of error cross-section independence. Econometrics Journal 11, 105-127 (2008).

Pesaran, M. H., Yamagata, T.: Testing slope homogeneity in large panels. Journal of Econometrics 142, 50-93 (2008).

Pesaran, M.H.: General diagnostic tests for cross section dependence in Panels. Cambridge Working Papers in Economics No. 0435, Faculty of Economics, University of Cambridge (2004).

Pouris, A. and Pouris, A.: The state of science and technology in Africa (2000-2004): A scientometric assessment. International Journal of Scientometrics 79:297-309 (2009). 
Price, D.S. Toward a model for science indicators. In Elkana, Y., Lederber, G.J., Merton, R.K., Thackray, A., and Zuckerman, H. (Eds). Toward a metric of science: the advent of science indicators. New York: John Wiley \& Sons (1978).

Romer, P.M.: Increasing returns and long-run growth. Journal of Political Economy 94:1002-1037 (1986).

Schubert, A. and Telcs, A.: Publication potential- An indicator of scientific strength for cross-national comparisons. Scientometrics 9:231-238 (1986).

Schumpeter, J.A.: Entrepreneurship as innovation. Entrepreneurship: The social science view 51-75. (Available at: http://ssrn.com/abstract=1512266) (2000).

Shelton, R.D. \& Leydesdorff, L.: Bibliometric Evidence for Funding Trade-Offs in National Funding Strategies, 13th International Conference on Scientometrics and Informetrics, Durban, July. 2011. Available at http://itri2.org/s/ (2011).

Swamy, P.A.V.B.: Efficient inference in a random coefficient regression model. Econometrica, 38, 311-323 (1970).

Tamura, R.F.: Fertility, human capital and the "Wealth of Nations". University of Iowa, Department of Economics. Working paper No 91-17 (1991).

Wharton (n.d.): What's driving India's rise as an R\&D Hub?. Knowledge@ Wharton, University of Pennsylvania. Available at http://knowledge.wharton.upenn.edu/special_section.cfm?specialID $=40$ 